\title{
Electronic Properties of Single-Walled Carbon Nanotube Networks
}

Elena Bekyarova, Mikhail E. Itkis, Nelson Cabrera, Bin Zhao, Aiping Yu, Junbo Gao,

$$
\text { Robert C. Haddon* }
$$

Center for Nanoscale Science and Engineering, and

Departments of Chemistry and Chemical \& Environmental Engineering

University of California, Riverside, California 92521

*corresponding author: robert.haddon@ucr.edu

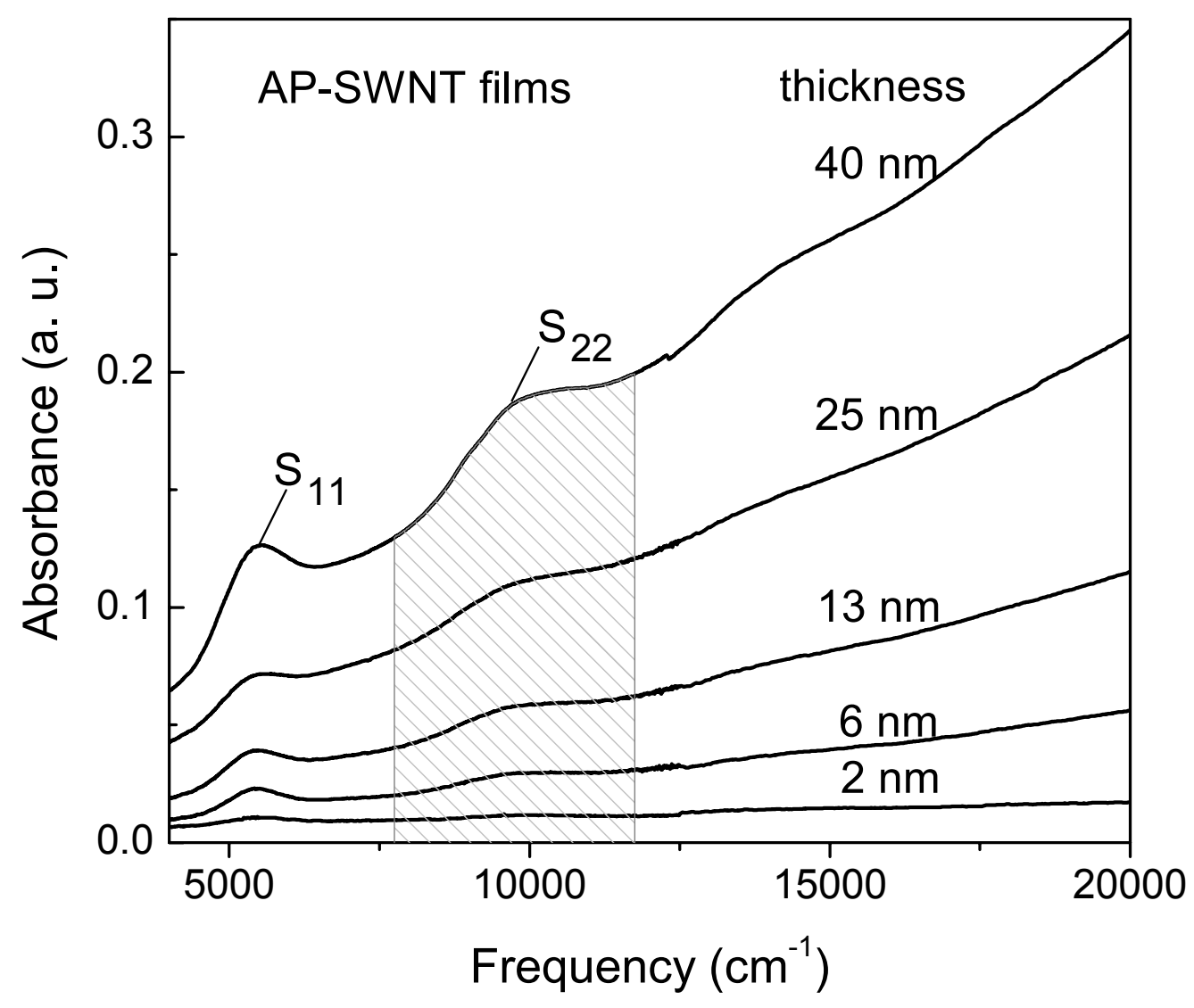

Figure S1. Absorption spectra of AP-SWNT films of various thicknesses. 


\section{Supporting Information}

Film thickness, $\mathrm{nm}$

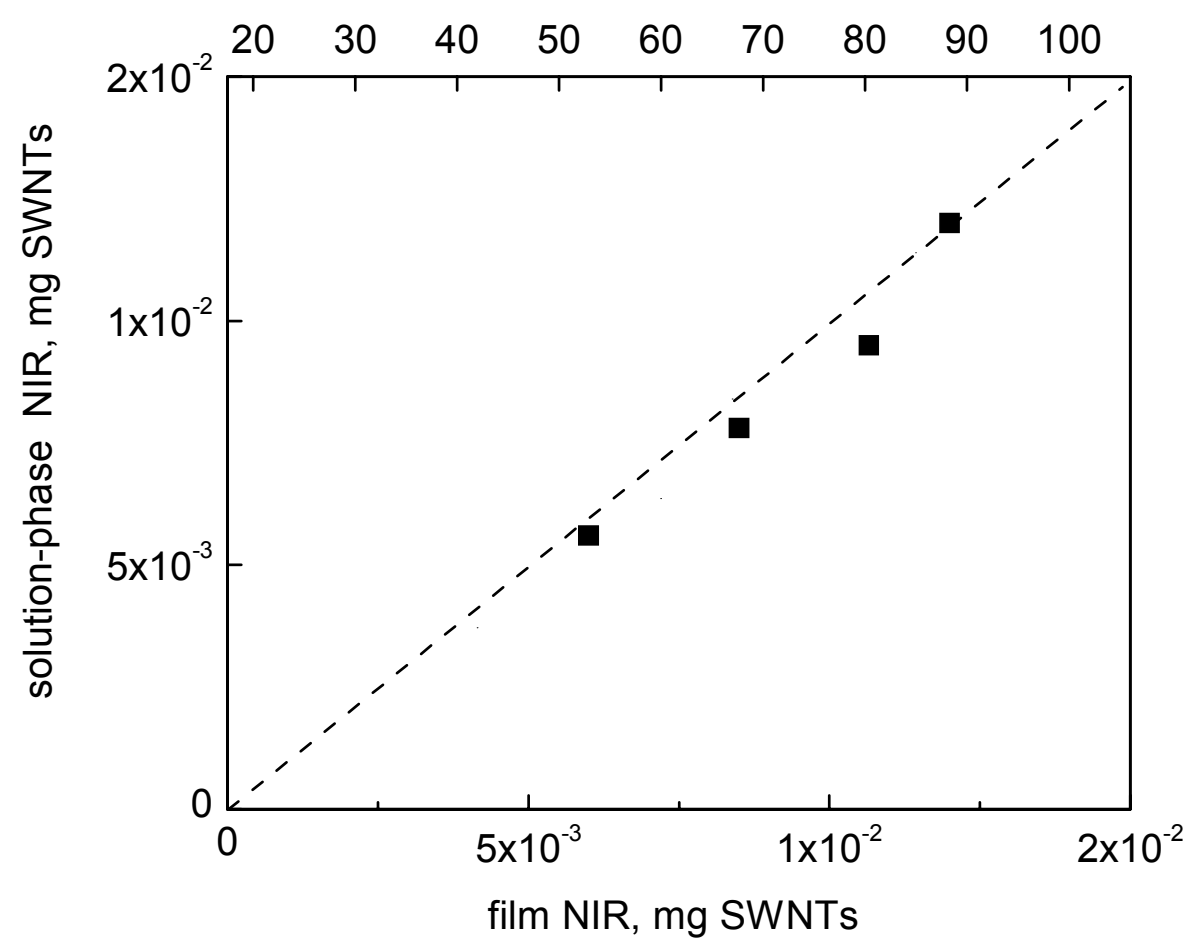

Figure S2. Amount of SWNTs used for the preparation of films of different thicknesses estimated from the second interband transition $\left(\mathrm{S}_{22}\right)$ of the near-IR (NIR) spectra of films and solutions. The solutions were obtained by redispersing the films in dimethylformamide. The dashed line corresponds to the exact match of the values obtained from the two methods (shown for clarity). 


\section{Supporting Information}

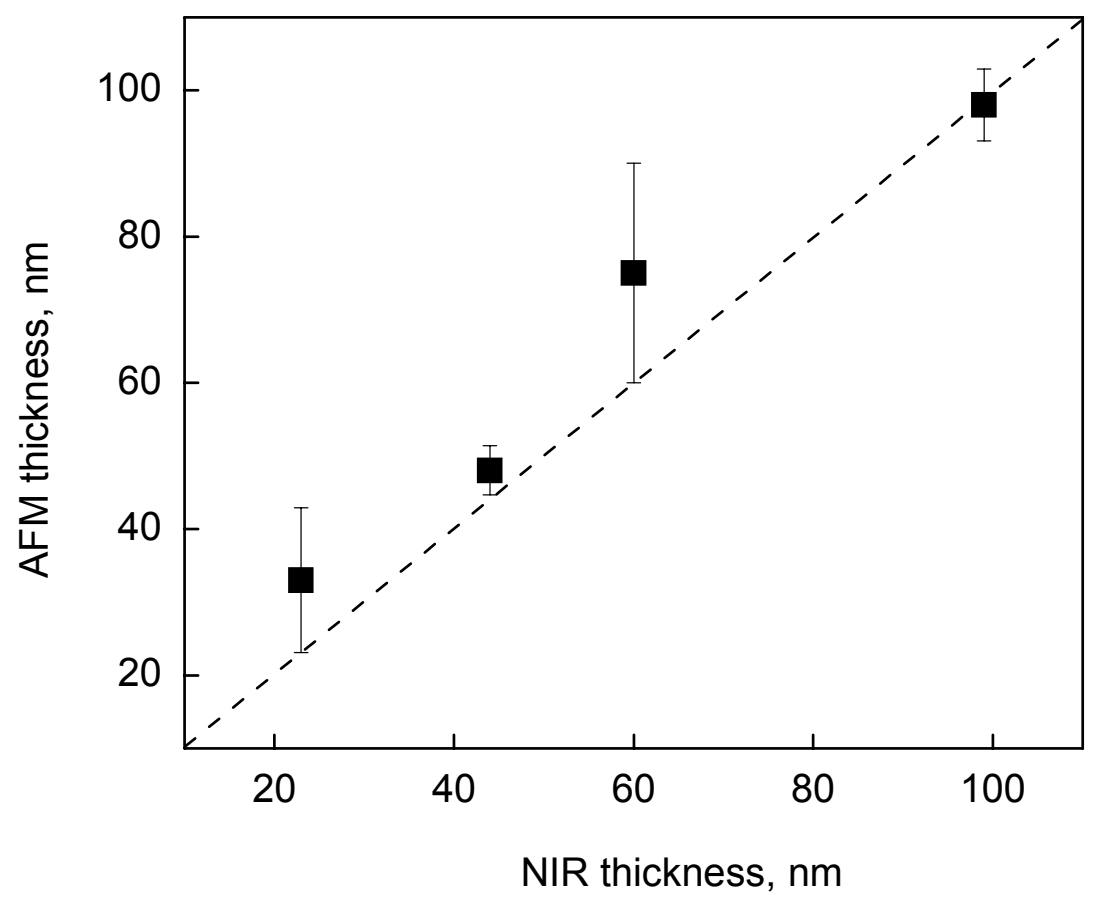

Figure S3. Thickness of SWNT films estimated by AFM and near-IR (NIR) spectroscopy. The AFM images were recorded at the film edge. The AFM data show significant scattering due to the film roughness. The dashed line corresponds to the exact match of the values obtained from the two methods (shown for clarity). 
Supporting Information
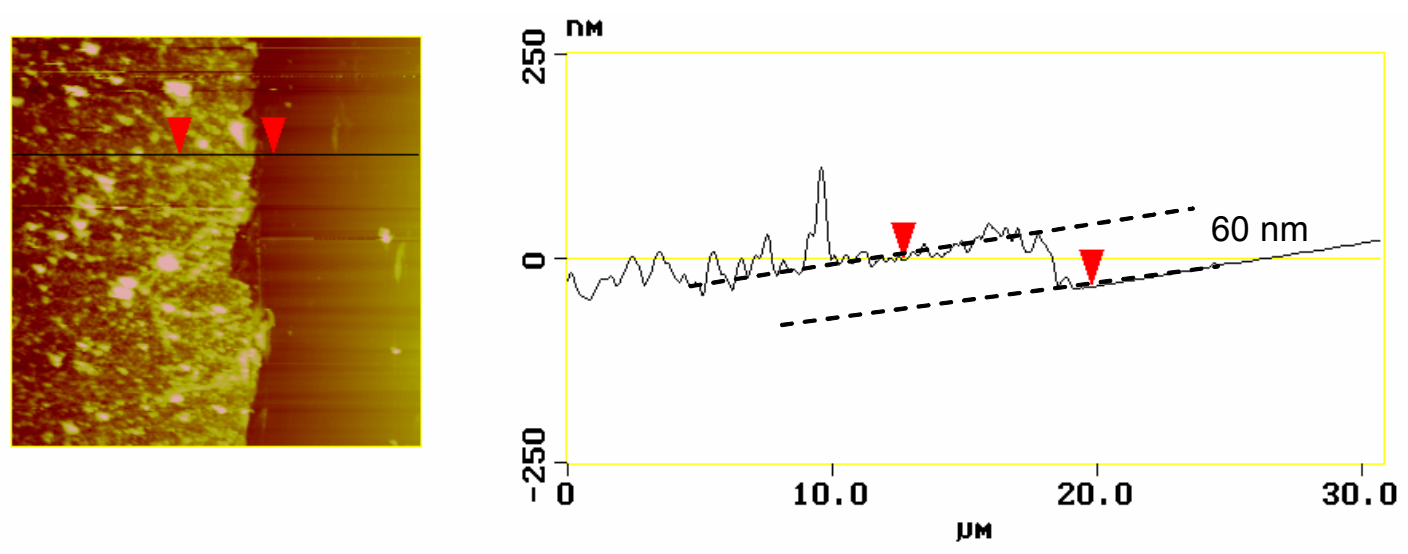

Figure S4. AFM analysis of film thickness for a $60 \mathrm{~nm}$ thick film. 